\title{
Is Gun Violence Contagious? A Spatiotemporal Test
}

\author{
Charles Loeffler (University of Pennsylvania), Seth Flaxman (University of Oxford)
}

July 27,2017

\begin{abstract}
Existing theories of gun violence predict stable spatial concentrations and contagious diffusion of gun violence into surrounding areas. Recent empirical studies have reported confirmatory evidence of such spatiotemporal diffusion of gun violence. However, existing space/time interaction tests cannot readily distinguish spatiotemporal clustering from spatiotemporal diffusion. This leaves as an open question whether gun violence actually is contagious or merely clusters in space and time. Compounding this problem, gun violence is subject to considerable measurement error with many nonfatal shootings going unreported to police. Using point process data from an acoustical gunshot locator system and a combination of Bayesian spatiotemporal point process modeling and classical space/time interaction tests, this paper demonstrates that contemporary urban gun violence in a metropolitan city does diffuse in space and time, but only slightly, suggesting that a disease model for the spread of gun violence in space and time may not be a good fit for most of the geographically stable and temporally stochastic process observed.
\end{abstract}

\section{Introduction}

Beginning with Loftin (1986), scholars of crime have described homicide in general, and gun-related homicide in particular as diffusion processes with contagion or epidemic properties (Reiss et al., 1993; Fagan et al., 2007; Patel et al., 2012). Within this paradigm, a variety of different contagion theories have been proposed, all positing that acts of violence increase the odds of further violence, though they differ in the mechanism and time-scale of violence transmission. Early work by Blumstein (1995) subsequently expanded by Fagan et al. (2007) articulated a macro-historical version of contagion theory wherein changing illegal markets led to multi-year increases in gun violence. Other scholars have suggested that violence propagates through social networks of connected individuals (Papachristos et al., 2016). Most scholars, however, have theorized contagion on a much shorter time scale with individual incidents leading to elevated risk of retaliatory shootings concentrated in the communities and lives of individuals connected to earlier incidents. This criminological and public health perspective, beyond its importance to the discipline, also provides a theoretical foundation for policy interventions such as violence interruption programs (Skogan et al., 2009; Webster et al., 2012), designed to disrupt the transmission of gun violence between specific individuals and within particular gun violence hotspots.

Empirical studies of the spatiotemporal properties of crime have reported robust evidence of space-time clustering of property crime (Johnson, 2008; Mohler et al., 2011; Short et al., 2010; Townsley et al., 2003) and violent crime (Cohen and Tita, 1999; Morenoff et al., 2001; Tita and Cohen, 2004; Ratcliffe and Rengert, 2008; Braga et al., 2010; Rosenfeld et al., 1999). Cohen and Tita (1999) and Tita and Cohen (2004) report evidence of spatial spillover of homicide and shots fired from census tracts with increasing rates to adjacent tracts, but only during peak crime periods. Messner et al. (1999) report non-random clustering of homicides at the county level, and Ratcliffe and Rengert (2008) reports evidence of a non-random spatiotemporal clustering at the block-level. Both interpret non-random space/time clustering as potential evidence of violence diffusion. However, scholars have yet to determine whether gun violence actually diffuses in space and time, consistent with an epidemic or similar contagion process, or merely clusters in space and time, consistent with endemic gun violence concentrated in certain locations at certain times. This limitation is due, at least in part, to the fact that existing space/time tests 
were designed to detect departures from complete spatiotemporal randomness rather than distinguishing between different types of non-random clustering.

Given the high levels of observed gun violence in many U.S. cities (Braga et al., 2010), including retaliatory shootings embedded in social networks that are themselves embedded in neighborhoods characterized by concentrated poverty (Morenoff et al., 2001; Tita and Ridgeway, 2007; Papachristos, 2009), gun violence could conceivably become contagious with violence triggering more violence (Fagan et al., 2007; Patel et al., 2012). Lending empirical support for this possibility, other human behaviors, including self-directed violence, have been shown to diffuse readily (Tarde, 1903; Coleman et al., 1957; Christakis and Fowler, 2007) and evidence of diffusion of violence in social networks has been recently reported (Rosenfeld et al., 1999; Papachristos, 2009; Short et al., 2014; Papachristos et al., 2016; Green et al., 2017).

At the same time, detailed examinations of homicide circumstances and motivations have revealed that much fatal gun violence is spontaneous (Metropolitan Police Department, 2006; Philadelphia Police Department, 2014; Chicago Police Department, 2012), resulting from arguments and failed drug transactions (See also Tita et al. (2003). The existence of non-retaliatory shootings alone, if they were randomly distributed in space-time, would simply add noise to estimates of the diffusion of any retaliatory shootings. However, if a sufficient fraction of all gun discharges in a city result from non-retaliatory shootings that are themselves clustered in space-time, then endemic gun violence with little or no diffusion in space or time could be confused for an epidemic or infectious process using conventional space/time interaction tests. ${ }^{1}$

Distinguishing between these two interpretations is important on both theoretical and policy grounds. Theoretically, diffusing gun violence would provide support for models of gun violence that emphasize its contagious/infectious features and suggest the need for additional studies focusing on the exact individual-level and mobility-based mechanisms through which elevated risk is transmitted through space and time. Non-random clustering without diffusion, on the other hand, highlights the role of social structure and place in determining the observed distributions. Similarly, the policy implications of these two interpretations can be quite different. Diffusion models have recently formed the theoretical basis for violence interruption programs targeting individuals at heightened risk of perpetration or victimization. Non-diffusion models, by contrast, suggest the need for placebased interventions that address persistent underlying structures (Braga, 2005; Weisburd et al., 2012)

To test these alternative possibilities and to more precisely describe the spatiotemporal point process of contemporary gun violence, we examine a dataset composed of data from an acoustic gunshot locator system (AGLS) installed in Washington, D.C, a city with a long history of gang and gun violence. Using this data, which overcomes the incomplete measurement of gun violence in conventional reported crime data, and refined space-time interaction tests, we observe that while gun violence does diffuse over space and time, this diffusion is quite minimal-limited in space to 126 meters and in time to 10 minutes-and thus much more likely to be consistent with a discrete gun fight, lasting for a matter of minutes, than with a diffusing, "infectious" process linking violent events across hours, days, or weeks. We then replicate these results using conventional calls for service (CFS) data on reported gunfire incidents. As such, these results provide little support for contagion models of violence diffusion in space and time; instead, this finding supports models predicting stochastic space/time clustering of gun violence (Braga et al., 2010).

\section{Methods}

At its most basic level, gun violence is an example of a spatiotemporal point process. Individual gunshots occur at specific geographic coordinates and at specific times. As such, gun violence lends itself to analysis using one of the several space-time interaction tests that have been developed to assess whether a point process is consistent with the random allocation of points across time and space or is sufficiently clustered that further investigation is warranted. One of the earliest space-time interaction tests, proposed by Knox $(1964 a, b)$, examines the distribution

\footnotetext{
${ }^{1}$ Previous typologies of gun violence (Fagan et al., 2007) use the term epidemic to describe both clustered gun violence with diffusion and clustered gun violence without diffusion. Since each of these two types of clustered gun violence are potentially the product of different mechanisms, for clarity, we refer to diffusing gun violence as epidemic and non-diffusing gun violence as endemic (Christoffel, 2007).
} 
of spatiotemporal distances between all points, reducing this combinatorial set of pairwise relationships to a simple $2 \times 2$ contingency table of near and far distances in both dimensions. Too many points in the near time and space cell (compared to a null model of independence) indicates clustering, which is not consistent with a homogeneous Poisson process. Ratcliffe and Rengert (2008) recently analyzed Philadelphia shooting data using this test and observed that an elevated number of shootings was present within two weeks and one block of a prior shooting.

Tests for space-time interaction like the Knox test are really testing for spatiotemporal clustering (Mantel, 1967), yet spatiotemporal clustering could have various explanations. Gunfire that occurs mostly on weekend nights in high crime neighborhoods, for instance, would produce reliable spatiotemporal clustering despite the fact that the spatial and temporal processes generating the shootings are independent. The difficulty of distinguishing between a non-diffusing but heterogeneous point process such as this and a diffusing point process in which each new point is potentially the parent of additional points in its immediate vicinity was identified as early as Bartlett (1964). More recently, Diggle et al. (2013) reiterated the importance and the difficulty of separating these two types of processes in a range of applications where stable and segregated point processes suggest substantially different theoretical mechanisms.

Up until recently, scholars in criminology and spatial statistics have used non-parametric descriptive statistics to assess the departures from randomness observed in the spatiotemporal crime processes (Johnson and Bowers, 2004; Johnson et al., 2007). For example, Wooditch and Weisburd (2015), demonstrated the uses of Ripley's KFunction for simultaneously examining space and time in program evaluations of stop and frisk in New York City. However, non-parametric spatial statistics have only recently been adapted to handle inhomogeneous underlying intensities, which are the norm in criminological applications. And it remains an open question whether these methods will prove robust enough to handle the typical criminological case in which the underlying intensity of crime is unknown. For this reason, scholars in a number of fields have adapted model-based spatiotemporal methods for use in characterizing spatiotemporal processes with both underlying intensities and conditional intensities. In work on crime, Mohler and colleagues were the first to demonstrate the utility of these models to the problem of predicting spatiotemporal point processes (Mohler et al., 2011). They showed that the Hawkes process model with its two components could readily fit the heterogeneous spatiotemporal point process of crime in urban settings while allowing for near-repeats events in the vicinity of high intensity property crimes. More recently, Meyer et al. (2016), showed that these same methods could serve to characterize a range of background and conditional intensities, in their application, hospital admissions. Building on these earlier efforts, we sought to adapt these methods for the problem of separating inhomogeneous background intensities from conditional intensities.

Our model Modern spatiotemporal point process models and increasingly fine-grained spatiotemporal data allow us to distinguish between spatiotemporal clustering and diffusion. To do this, we employ a Hawkes process model. Given a set of observations indexed by their space-time locations, $\left(x_{1}, y_{1}, t_{1}\right), \ldots,\left(x_{n}, y_{n}, t_{n}\right)$, the conditional intensity of the Hawkes process is given by:

$$
\lambda(x, y, t)=m_{0} \cdot \mu(x, y, t)+\theta \sum_{i: t_{i}<t} \omega \exp \left(-\omega\left(t-t_{i}\right)\right) \frac{1}{2 \pi \sigma^{2}} \exp \left(-\left(\left(x-x_{i}\right)^{2}+\left(y-y_{i}\right)^{2}\right) /\left(2 \sigma^{2}\right)\right)
$$

where we have specified an exponential form for the temporal triggering kernel and a Gaussian form for the spatial kernel following Mohler (2014). There are two components to this model, an underlying endemic spatiotemporally varying intensity $\mu(x, y, t)$ weighted by a non-negative parameter $m_{0}$ which we estimate and a self-excitatory conditional intensity that depends on the history of previous events. We perform Bayesian inference to infer posteriors over the parameters in the model. Priors and model implementation details are discussed in the Appendix in Section A.1.

Of particular interest are the posterior distributions over the lengthscale (bandwidth) parameters for the spatial and temporal kernels as these enable us to characterize the extent of diffusion in space and time. Also of interest is the excitation parameter $\theta$, which gives the average number of shootings triggered by any particular shooting. Finally, for a shooting at location $\left(x_{i}, y_{i}, t_{i}\right)$ we can calculate the ratio $r_{i}=m_{0} \cdot \mu\left(x_{i}, y_{i}, t_{i}\right) / \lambda\left(x_{i}, y_{i}, t_{i}\right)$. This ratio is the fraction of intensity explained by our model's endogeneous component for observation $i$, while $1-r_{i}$ 
is the fraction explained by the excitatory component. Some points will be better explained by the background process than others, and we explore how much variation there is in $r_{i}$ for $i=1, \ldots, n$.

To capture the first-order space/time clustering corresponding to the highly uneven distribution of gun violence in space (Braga et al., 2010) and time (Metropolitan Police Department, 2006; Philadelphia Police Department, 2014; Chicago Police Department, 2012), we use kernel intensity estimation to separately estimate $\hat{\mu}_{s}(x, y)$ and $\hat{\mu}_{t}(t)$, obtaining a separable estimator $\hat{\mu}(x, y, t)=\hat{\mu}_{s}(x, y) \hat{\mu}_{t}(t)$. This separable approach to estimating the underlying intensity follows Diggle et al. (2005) where it was used for an infectious disease modeled by a Cox process. We are protected against overfitting because $\hat{\mu}_{s}(x, y)$ and $\hat{\mu}_{t}(t)$ are estimated separately. We consider this to be a conservative approach to take, insofar as overfitting might lead us to miss a weak self-exciting signal if we were to incorrectly attribute it to the endemic background process. We note that while it might be reasonable to include covariates like socioeconomic indicators from the census in this estimation step, no covariates match the spatiotemporal resolution of our data, so we would not expect them to add any additional information. We use Epanechnikov kernels (Ramlau-Hansen, 1983) with relatively long spatial and temporal bandwidths. We perform a sensitivity analysis in the Appendix in Section A.4 showing that our results are robust to various choices of the bandwidth parameters of the smoothing kernel.

While this two-stage model necessarily involves more complexity than past space/time clustering methods such as the Knox or the K-function, the segmentation of clustering into first-order and second-order components offers a convenient metric for evaluating the relative importance of background and conditional intensities. This approach can also be thought of as a compromise between the non-model-based test statistics, which are ill-equipped to accommodate inhomogeneous backgrounds, and SIR models, which cannot easily be made to fit our setting, as there is no natural way of observing who within the population is susceptible to infection, infected, or recovered.

\section{Data}

Conventional crime data on gun violence is gathered from human-witnesses who notify police of fatal, non-fatal, and miss or near-miss shootings in their immediate vicinity. While reported shootings are generally thought to be a near-random subset of all discharges (Zimring, 1967), it is generally understood that no more than a quarter to a third of illegal firearm discharges are reported to police (Mares and Blackburn, 2012). Due to concerns about this "dark figure" of unreported gun crime, increasingly, municipalities with persistent gun violence problems are deploying acoustical gunshot locator systems (AGLS) to enable the detection of illegal gun discharges unreported to police through 911 systems.

A network of acoustical sensors, AGLS systems operate by recording the impulsive sounds of gun discharges and filtering out other impulsive sounds (e.g., jack-hammers, car-back-fires, fireworks) that might be confused with gunshot events. While military applications of this technology can use a combination of shockwave and muzzle blast features to detect and localize the point of origin of a ballistic projectile, the smaller caliber munitions commonly observed in domestic security applications preclude the use of the shockwave feature (Sallai et al., 2011). As a result, AGLS used in domestic public safety applications rely solely on time-of-arrival of the muzzle blast acoustical signal at multiple microphones arrayed at known distances. Using this information, the bullet origin can be computed using multilateration. While accuracy concerns remain, in practice, AGLS systems record between 2 and 4 times more firearm "shots fired" incidents than conventional 911 incident systems (Mares and Blackburn, 2012).

We use AGLS data from Washington, D.C. covering the period from January 1, 2010 to December 31, 2012. Sensor coverage during this period included parts of six of the D.C.'s seven police districts with greatest sensor concentration in the Northeast and Southeast quadrants. Gunshot event data were rounded to produce approximately $100 \mathrm{~m}$ spatial resolution and 1 second temporal resolution. For a detailed discussion of the DC deployment, see Petho et al. (2013). Shootings occurring less than 1 minute and $100 \mathrm{~m}$ in time and space were merged (See Appendix Section A.5).

Figure 1a indicates a high degree of temporal clustering around New Year's and July 4th. Removing these temporal clusters (see Appendix A.2) does little to reduce seasonal temporal clustering, since summer months 


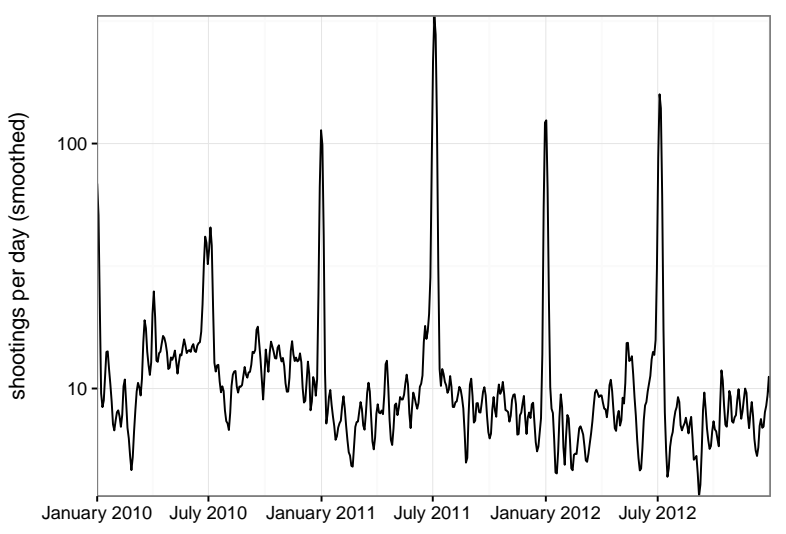

(a) Time series

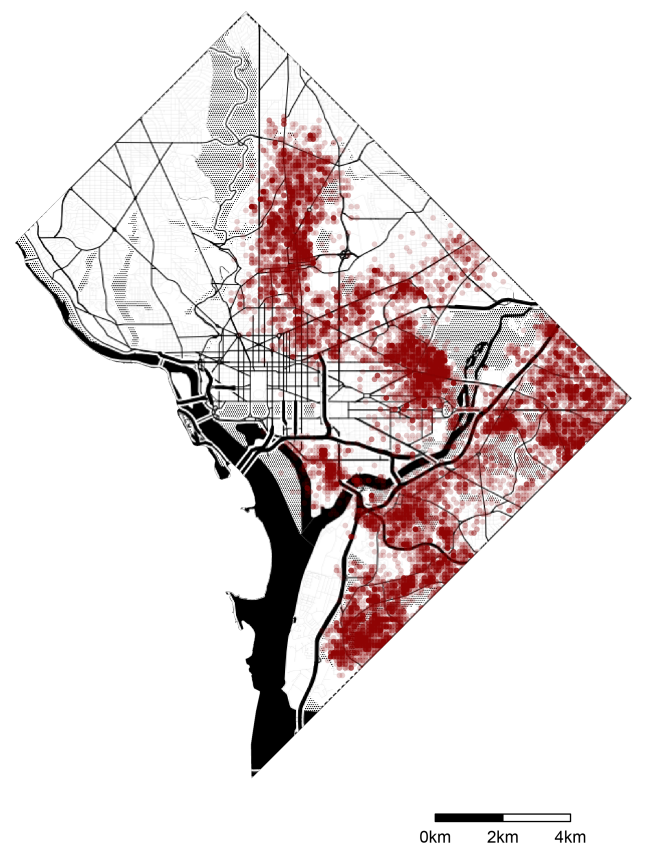

(c) AGLS

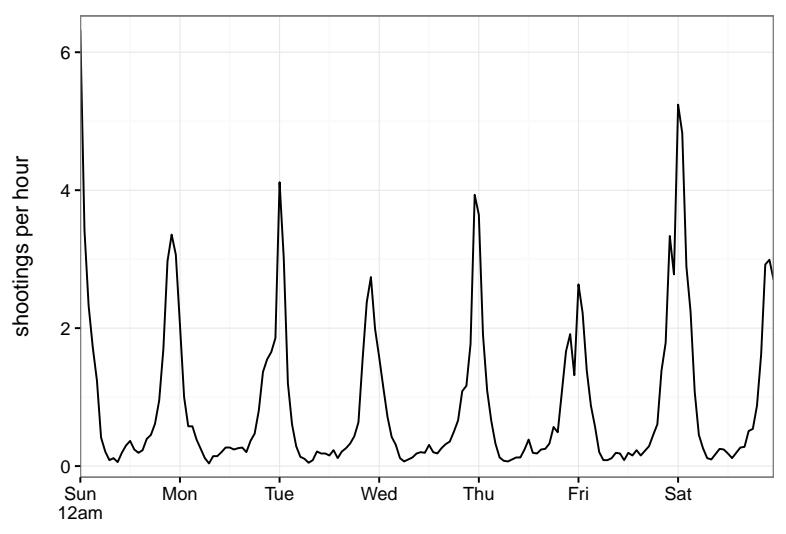

(b) Hour of week trends

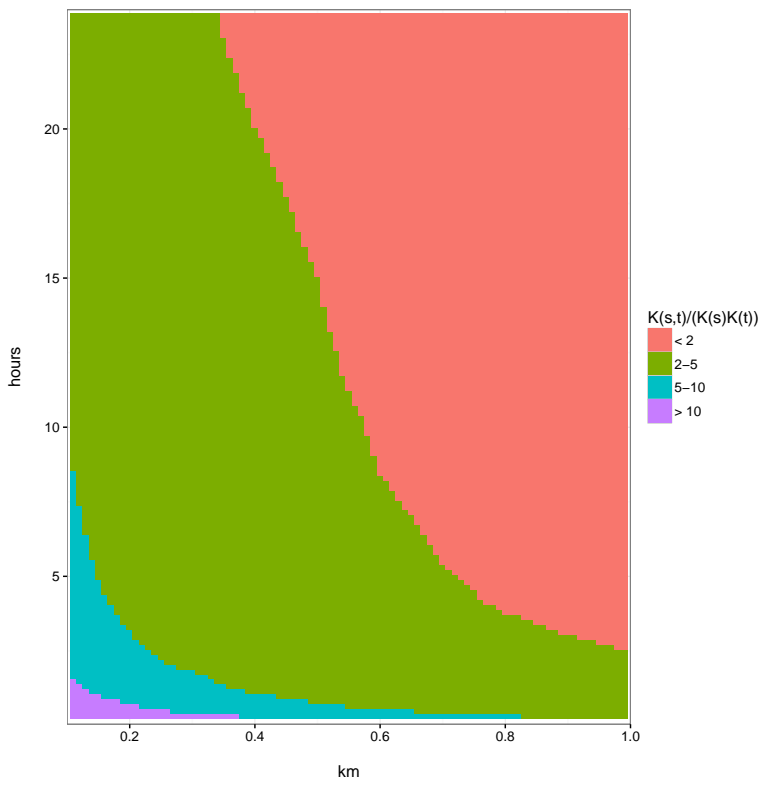

(d) Ripley's K-Function

Figure 1: (a-b). Temporal distribution of acoustically located gunshots in Washington D.C., 2010-2012. (c). Spatial distribution of AGLS, 2010-12. (d). Ripley's K-Function.

have much more activity than other months. High degrees of day-of-week and hour-by-hour periodicity can also be observed (Figure 1b). The spatial intensity of acoustically detected gunshots (Figure 1c) also demonstrates a high degree of spatial clustering. Noticeable concentrations can be observed in subsections of the southeast and northeast city quadrants. Adjacent areas appear to have sparser densities. 
Compared to conventional firearm use data, as seen in Appendix Figure 5c, the AGLS firearm data shows much higher densities of illegal firearm usage. For the city as a whole, during the years 2011 and 2012, police recorded 577 and 614 firearm assaults, respectively. By comparison, the AGLS system, despite incomplete coverage, recorded 6,668 and 5,385 gunshot events in 2011 and 2012, respectively. These striking level differences reflect both the considerable number of unreported discharges as well as the sizable number of miss events that did not result in physical injury. At the same time, both weekly time series are highly correlated, suggesting that despite their level differences, both measures are likely capturing a common quantity with differing amounts filtering.

This considerable amount of spatial and temporal clustering means that the null hypotheses for classical tests of space/time clustering will always be rejected. For the present application, following Diggle et al. (1995), we calculated Ripley's $\mathrm{K}$ function in space and time, $K(s, t)$ and divided it by the product of separate $\mathrm{K}$ functions $K(s) K(t) . K(s, t)$ is proportional to the expected number of additional points within a distance $s$ and time window $t$ of an arbitrary event (Cressie and Wikle, 2011, p. 210). By comparing $K(s, t)$ to $K(s) K(t)$ we are looking for excess gunshots as compared to how many we would expect based on the separable product of $K(s)$ and $K(t)$. Based on this analysis, space-time clustering is clearly evident (see Figure 1d). If firearm assault or homicide data approximate a random sample of all firearm discharges, this inhomogeneous clustering could be observed in conventional crime data as well. In the next section, we examine alternative explanations for this space/time clustering.

\section{Results}

We estimated the underlying spatiotemporal intensity by separately performing kernel intensity estimation in space and time, with a spatial bandwidth of 1.6 kilometers and a temporal bandwidth of 14 days. While a range of bandwidths were considered and tested, this bandwidth was learned through Bayesian inference. To perform Bayesian inference we used Markov Chain Monte Carlo (specifically HMC as implemented in Stan Stan Development Team (2016)), running 4 chains for 1000 iterations each, discarding 500 iterations as warmup, to obtain a total of 2000 draws from the posterior. Convergence diagnostics are in the Appendix in Section A.7.

The posterior estimates of the parameters in our model in Eq. (4) are as follows: $m_{0}$, the weight placed by our model on the underlying endogeneous intensity (as opposed to the triggered self-excitatory component) was 0.87 [95\% posterior credible interval $0.86,0.89]$. Correspondingly, the model estimated the parameter $\theta$, giving the average number of shootings triggered by a particular shooting, to be 0.13 [0.12, 0.14]. Intriguingly, this split (87/13) between endogeneous and self-excitatory shootings closely matches the reported split (86/14) between nonretaliatory and retaliatory shootings from examining homicide motives (Metropolitan Police Department, 2006).

The temporal lengthscale $1 / \omega$ for the exponential triggering kernel is estimated to be 10 minutes [9.5,11]. The spatial lengthscale $\sigma$ was estimated to be 126 meters [121, 133]. The excitatory component of Eq. (4) is visualized in Figure 2 where the extremely local ranges of the self-excitatory process are evident. At time 0 and spatial distance 0 , the excitatory component of the Hawkes process has a value of $178[157,200]$ shootings per $\mathrm{km}^{2}$-hour which equals $4.1[3.6,4.7]$ shootings per block-hour. Due to the extremely local range of the space and time kernels, the intensity drops off very quickly, so it is more intuitive to interpret to $\theta$.

We interpret $\theta=0.13$ as follows: if we were to observe 100 shootings at a given location (which would be many more than we expect), we would on average expect to see 13 additional shootings, very nearby in space and time (most within 11 minutes and 133 meters, using the upper end of the 95\% uncertainty interval). From those 13 additional shootings, we would expect to see be between 1 and 2 additional shootings, again very nearby in space and time. And so on, for a total of about 115 shootings. Allowing for the most liberal spatial and temporal extents, the 15 offspring shooting triggered by the original 100 shootings will occur within just half an hour and 500 meters. Since there were a total of 9,831 shootings in our dataset, we thus considered the 1,278 shootings with the highest estimated conditional intensity to be triggered. These shootings are highlighted in red, with background shootings in blue, in Figure 3.

To evaluate the fit of our model, we use it to make predictions of the number of shootings we expect to see in 1 


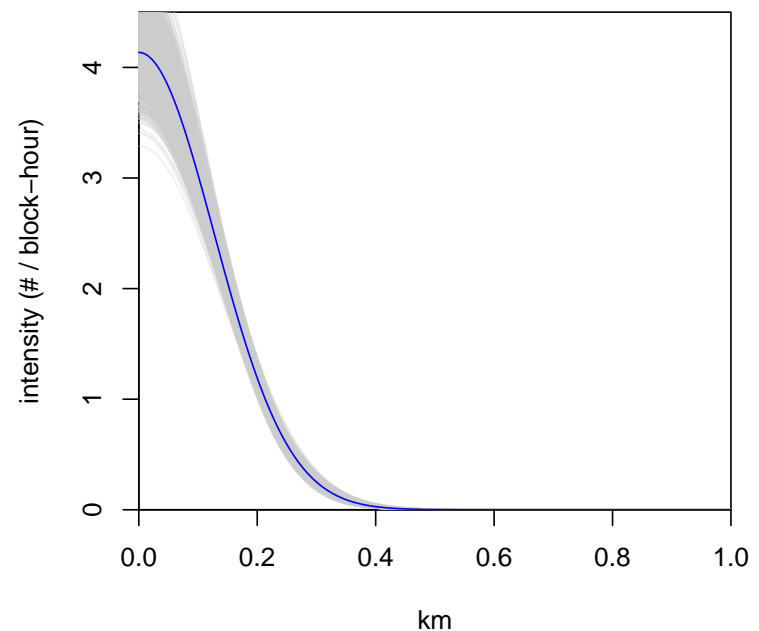

(a) Conditional intensity in space at time $t=0$

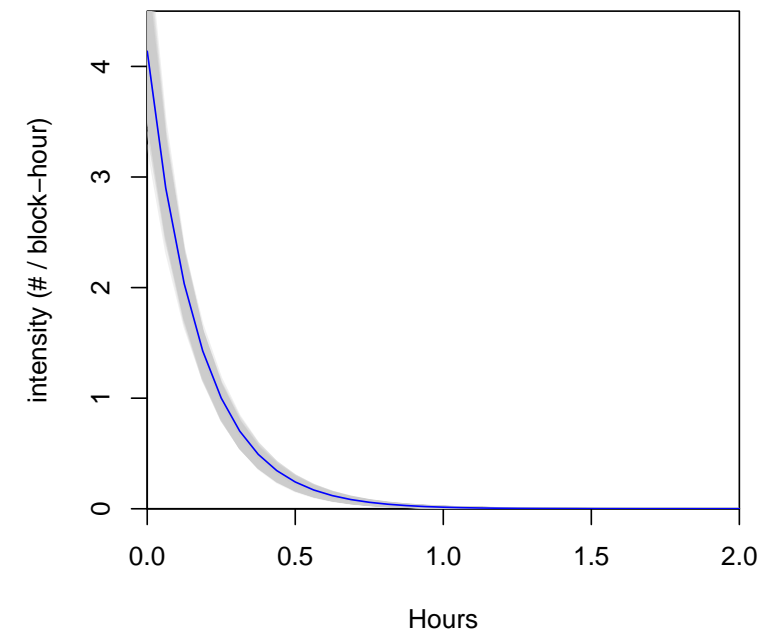

(b) Conditional intensity in time at distance $s=0$

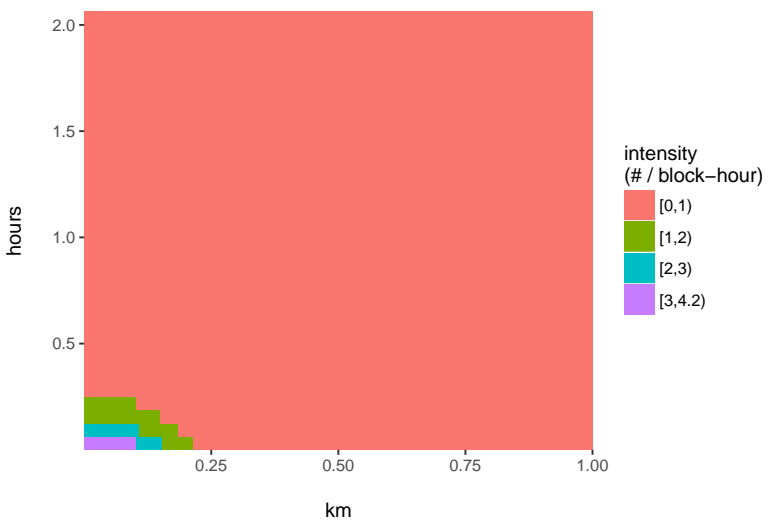

(c) Spatiotemporal conditional intensity

Figure 2: The self-excitatory component of the Hawkes process, corresponding to the product of a spatial and temporal kernel, is visualized in space (a) at time $t=0$, in time (b) at distance $s=0$, and in space/time (c). In (a) and (b) the blue line is the mean of the posterior distribution. The gray lines show samples from the posterior distribution, reflecting the (small amount) of posterior uncertainty in the parameter estimates.

$\mathrm{km}^{2}$ by 1 hour circles centered on a Cartesian grid spanning our obervations. On a given day this means we make $102 \times 24$ predictions. If we were to use just the kernel smoothed endogeneous intensity, the correlation between the observed number of shootings and the kernel smoothed intensity surface is 0.06 , which simply reflects the long lengthscales we chose for this smoothing. If we make predictions using the Hawkes process model that we fit, the correlation is 0.20 .

While there is nothing in our model specification favoring short spatial and temporal lengthscales, the posterior was quite concentrated, as shown in the Appendix in Figure 7. As a further check, we tried fixing the temporal lengthscale for the self-excitation process at a range of values, from 5 minutes to 180 days, and refitting the model 
so as to learn the other parameters, including the spatial lengthscale. We compared the mean of the posterior joint log-likelihood across this range of temporal lengthscales values. Short temporal lengthscales (under 2 hours) all had high likelihoods corresponding to our main result. For lengthscales 1,2, and 3 days the likelihood decreased significantly (i.e. the model became less likely) as the lengthscale increased. For lengthscales above 3 days, the model did not converge, with the learned spatial lengthscale diverging to zero.

As a check on our basic finding, we turn to the Knox test, a much simpler test for space/time interaction used in slightly modified form in Ratcliffe and Rengert (2008). Our results suggest that there this is very local and concentrated space/time clustering, so the Knox test should reject the null hypothesis of independence at any practical spatial and temporal bandwidths, and indeed for 134 meters and 11 minutes, the Knox test resulted in a p-value $\leq 0.001 .^{2}$

While AGLS data potentially improves on under-reporting found in conventional police data, it itself is subject to two potential problems. First, it may be more subject to false positives than 911 call data. Second, it is currently only available for a fraction of cities and communities that are experiencing gun violence problems. To verify that our spatiotemporal models could be used on 911 calls for service data, we refit our final model using the 911 calls for service data for "sounds of gunshots" for an overlapping temporal period beginning in 2011 and ending in 2013. The spatial lengthscale was 221 [214,228] meters. The temporal lengthscale was 9 [8.4,9.4] minutes. And the self-exciting paramters, corresponding to the fraction of events attributable to the conditional as opposed to background intensity, was $0.15[0.14,0.16]$. These results closely matched those obtained using AGLS data. The degree of similarity is likely a product of the high fraction of gunshot events reported to police both by civilians and the AGLS system in Washington, DC. In cities with more limited reporting or deployment, models fit on different data sources may not yield as consistent results.
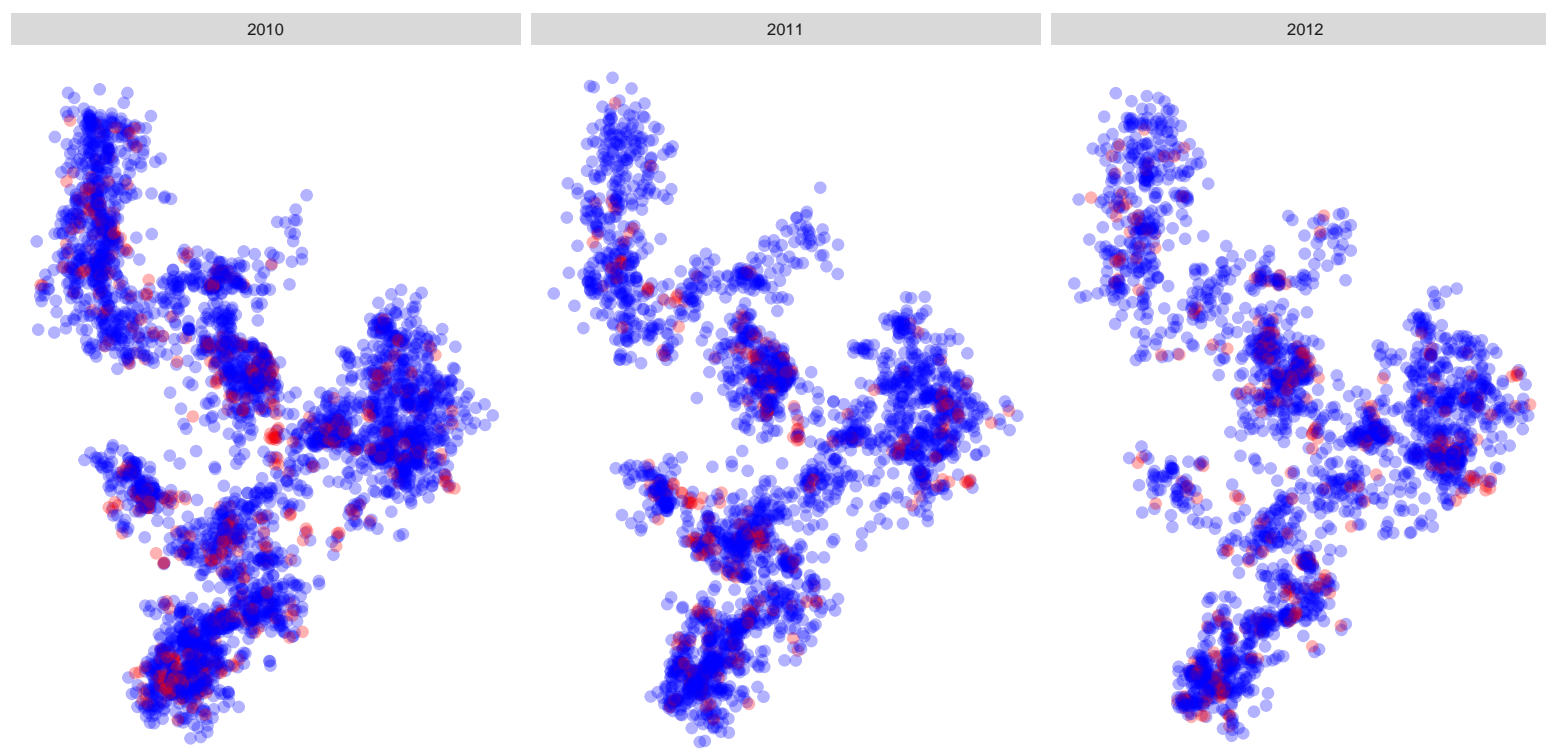

Figure 3: Background (blue) versus "triggered" (red) shootings, by year. At any location in space-time, our inferred model yields a posterior distribution over the intensity at that location. For each shooting in our dataset, we calculated the excitatory intensity (see Eq. (4)) as a way of classifying shootings as either coming from a background process or as being "triggered" by previous shootings. Based on the parameter $\theta$ in our model, $13 \%$ of shootings were caused by previous shootings, so we classified as "triggered" (red) shootings that fell in the top 13th percentile of excitatory intensity.

\footnotetext{
${ }^{2}$ Johnson and Bowers (2004); Johnson et al. (2007) used a non-cumulative Knox test in analyzing burglary data.
} 


\section{Discussion}

Existing theories of gun violence predict stable spatial concentrations and widespread diffusion of gun violence into surrounding communities, which should, in principle, make gun violence susceptible to a range of interventions. While these theories were originally developed to explain multi-year changes in levels of gun violence (Blumstein, 1995; Christoffel, 2007), they have recently been adapted to explain the patterns of gun violence observed within communities on a much shorter time-scale (Fagan et al., 2007). Past research, however, has yet to show that gun violence diffuses in either space or time due in part to the space-time interaction tests employed, which have not allowed for a characterization of the extent of any departure from complete spatiotemporal randomness in the space-time distribution of gun violence. The present investigation addressed this limitation by employing space-time interaction models that support a separation of space-time interactions consistent with epidemic or contagion-like diffusion processes from more conventional and constrained clustered distributions. Based on these new methods, it appears that while contemporary gun violence does diffuse in both space and time, this diffusion is quite minimal with spatial diffusion limited to just 126 meters and temporal diffusion to ten minutes after an initial shooting incident.

Given the many studies of gun violence that have reported evidence of retaliatory shootings as well as other forms of gun violence diffusion in space/time, it is worth considering why there is so little evidence of spatiotemporal gun violence contagion in the present study. One possible explanation is that previous analyses have been unable to separate highly clustered space-time violence into endemic and epidemic components due to the use of spatiotemporal statistics designed only to separate space-time clustering from complete spatiotemporal randomness. Another possible explanation is that in the more complete distribution of gun violence observed in this study, gun violence is both more spontaneous and less reactive than in the subset of shootings that result in a homicide or near homicide. A final possibility is that most observed gun violence is spontaneous rather than planned.

Lending support for this final possibility are recent examinations of perpetrator motives in a number of cities (Metropolitan Police Department, 2006; Philadelphia Police Department, 2014; Chicago Police Department, 2012), which show that most shootings result from arguments and failed drug transactions, and are therefore more likely than retaliatory shootings to be self-extinguishing. This appears to be the case for Washington, D.C., where retaliatory gun violence appears to be a less frequent explanation for gun-related homicides (Metropolitan Police Department, 2006). Whether or not other cities such as Los Angeles or Chicago, with larger and more established gangs, follow this pattern perfectly, it is worth recognizing that even in these cities a large fraction of gun violence is likely to be spontaneous. Similarly, to the extent that gang-related violence in other cities fosters retaliatory violence that diffuses through social networks rather than euclidean space, a possibility considered in several recent papers (Papachristos et al., 2016; Green et al., 2017), future work will likely need to confront the equivalent problem-separating non-random network clustering from non-random network diffusion (Shalizi and Thomas, 2011). Finally, macro-historical or cultural diffusion theories of violence, which predict an equilibrium change in the acquisition or utilization of firearms accompanying changes in illegal markets or social structure, will require somewhat different empirical tests that can adequately account for the multi-year time scale at which these mechanisms are theorized to operate (Blumstein, 1995; Hemingway, 2004; Fagan et al., 2007).

Focusing solely on contemporary gun violence, non-random clustering and stable violence hotspots have both been previously reported (Ratcliffe and Rengert, 2008; Braga et al., 2010). The stability of these clusters, some lasting decades, suggest that while macro-historical changes in culture, weapon-carrying, and illegal markets may help explain why these hotspots emerged, theories focused on shorter time scale processes will likely be necessary to explain their persistence as well as their intermittency. Contagion theories that highlight retaliatory violence provide one such explanation-victimization breeds retaliation. Alternatively, the processes of fear of victimization, under-policing, illegal markets, could create a structural setting in which many individuals are armed or nearly armed in moments that often give rise to spontaneous conflict. While both explanations would predict the observed clustering of gun violence in space/time, they predict different versions. One which includes strategic-decisionmaking and one which accommodates a more accidental process for generating the nearly identical non-random clustering pattern.

Taken together, these results suggest that for cities like Washington the reality of gun violence is only partially 
described by a theoretical focus on retaliatory gun violence. And that the model or metaphor of violence as a contagion may foster a misimpression (Dodge, 2008), since it deemphasizes evidence of non-diffusing clustering observed. Instead, the largely stable and stochastic space/time distributions observed reinforces the importance of place in understanding contemporary gun violence (Branas et al., 2017). This question of emphasis is not simply an academic matter. Place-based structural factors have been shown repeatedly to be important determinants of crime and violence in communities (Weisburd et al., 2012). And if gun violence is primarily place-based, then interventions targeting place offer a logical policy response alongside policies targeting other pathways to gun violence (Branas et al., 2011). ${ }^{3}$ Given the seeming substitutability of AGLS and CFS data, future work could usefully apply the methods described in this paper across a wider range of cities. Doing so could shed additional light on the relative importance of these two different models of gun violence and the usefulness of their two corresponding policy prescriptions for a broader array of cities and settings.

\footnotetext{
${ }^{3}$ Interestingly, a recent hotspot experiment suggests that the distinction between place-based and offender-based interventions may not always hold in practice (Groff et al., 2015).
} 


\section{References}

Bartlett, M. S. (1964), 'The Spectral Analysis of Two-Dimensional Point Processes', Biometrika 51(3/4), 299-311.

Blumstein, A. (1995), 'Youth Violence, Guns, and the Illicit-Drug Industry', The Journal of Criminal Law and Criminology (1973-) 86(1), 10-36.

Braga, A. A. (2005), 'Hot spots policing and crime prevention: A systematic review of randomized controlled trials', Journal of Experimental Criminology 1(3), 317-342.

Braga, A. A., Papachristos, A. V. and Hureau, D. M. (2010), 'The Concentration and Stability of Gun Violence at Micro Places in Boston, 19802008', Journal of Quantitative Criminology 26(1), 33-53.

Branas, C. C., Cheney, R. A., MacDonald, J. M., Tam, V. W., Jackson, T. D. and Ten Have, T. R. (2011), 'A Difference-in-Differences Analysis of Health, Safety, and Greening Vacant Urban Space', American Journal of Epidemiology .

Branas, C. C., Jacoby, S. and Andreyeva, E. (2017), 'Firearm violence as a diseasehot people or hot spots?', JAMA internal medicine 177(3), 333-334.

Chicago Police Department (2012), 2011 Chicago Murder Analysis, Technical report, City of Chicago, Chicago, IL.

Christakis, N. A. and Fowler, J. H. (2007), 'The Spread of Obesity in a Large Social Network over 32 Years', New England Journal of Medicine 357(4), 370-379.

Christoffel, K. K. (2007), 'Firearm injuries: epidemic then, endemic now', American journal of public health 97(4), 626-629.

Cohen, J. and Tita, G. (1999), 'Diffusion in Homicide: Exploring a General Method for Detecting Spatial Diffusion Processes', Journal of Quantitative Criminology 15(4), 451-493.

Coleman, J., Katz, E. and Menzel, H. (1957), 'The Diffusion of an Innovation Among Physicians', Sociometry 20(4), 253-270.

Cressie, N. and Wikle, C. (2011), Statistics for spatio-temporal data, Vol. 465, Wiley.

Diggle, P. J., Chetwynd, A. G., Hggkvist, R. and Morris, S. E. (1995), 'Second-order analysis of space-time clustering', Statistical Methods in Medical Research 4(2), 124-136.

Diggle, P. J., Moraga, P., Rowlingson, B. and Taylor, B. M. (2013), 'Spatial and Spatio-Temporal Log-Gaussian Cox Processes: Extending the Geostatistical Paradigm', Statistical Science 28(4), 542-563.

Diggle, P., Rowlingson, B. and Su, T.-1. (2005), 'Point process methodology for on-line spatio-temporal disease surveillance', Environmetrics 16(5), 423-434.

Dodge, K. A. (2008), 'Framing Public Policy and Prevention of Chronic Violence in American Youths', The American psychologist 63(7), 573-590.

Fagan, J., Wilkinson, D. L. and Davies, G. (2007), Social Contagion of Violence, in D. J. Flannery, A. T. Vazsonyi and I. D. Waldman, eds, ‘The Cambridge Handbook of Violent Behavior and Aggression', Cambridge University Press, New York, pp. 688-726.

Gelman, A., Jakulin, A., Pittau, M. G. and Su, Y.-S. (2008), 'A weakly informative default prior distribution for logistic and other regression models', The Annals of Applied Statistics pp. 1360-1383. 
Green, B., Horel, T. and Papachristos, A. V. (2017), 'Modeling contagion through social networks to explain and predict gunshot violence in chicago, 2006 to 2014', JAMA internal medicine .

Groff, E. R., Ratcliffe, J. H., Haberman, C. P., Sorg, E. T., Joyce, N. M. and Taylor, R. B. (2015), 'Does what police do at hot spots matter? the philadelphia policing tactics experiment', Criminology 53(1), 23-53.

Hemingway, D. (2004), Private Guns, Public Health, University of Michigan Press.

Johnson, S. D. (2008), 'Repeat burglary victimisation: a tale of two theories', Journal of Experimental Criminology 4(3), 215-240.

Johnson, S. D., Bernasco, W., Bowers, K. J., Elffers, H., Ratcliffe, J., Rengert, G. and Townsley, M. (2007), 'Spacetime patterns of risk: a cross national assessment of residential burglary victimization', Journal of Quantitative Criminology 23(3), 201-219.

Johnson, S. D. and Bowers, K. J. (2004), 'The Stability of Space-Time Clusters of Burglary', British Journal of Criminology 44(1), 55-65.

Knox, E. G. (1964a), 'The Detection of Space-Time Interactions', Journal of the Royal Statistical Society. Series C (Applied Statistics) 13(1), 25-29.

Knox, G. (1964b), 'Epidemiology of Childhood Leukaemia in Northumberland and Durham', British Journal of Preventive and Social Medicine 18(1), 17-24.

Loftin, C. (1986), 'Assaultive violence as a contagious social process.', Bulletin of the New York Academy of Medicine 62(5), 550-555.

Mantel, N. (1967), 'The Detection of Disease Clustering and a Generalized Regression Approach', Cancer Research 27(2 Part 1), 209-220.

Mares, D. and Blackburn, E. (2012), 'Evaluating the Effectiveness of an Acoustic Gunshot Location System in St. Louis, MO', Policing 6(1), 26-42.

Messner, S. F., Anselin, L., Baller, R. D., Hawkins, D. F., Deane, G. and Tolnay, S. E. (1999), 'The Spatial Patterning of County Homicide Rates: An Application of Exploratory Spatial Data Analysis', Journal of Quantitative Criminology 15(4), 423-450.

Metropolitan Police Department (2006), A Report on Juvenile and Adult Homicide in the Distict of Columbia, Technical report, Metropolitan Police Department, Research and Resource Development Department, Washington, D.C.

Meyer, S., Warnke, I., Rossler, W. and Held, L. (2016), 'Model-based testing for space-time interaction using point processes: An application to psychiatric hospital admissions in an urban area', Spatial and Spatio-temporal Epidemiology 17, 15-25.

Mohler, G. (2014), 'Marked point process hotspot maps for homicide and gun crime prediction in chicago', International Journal of Forecasting 30(3), 491 - 497.

Mohler, G. O., Short, M. B., Brantingham, P. J., Schoenberg, F. P. and Tita, G. E. (2011), 'Self-Exciting Point Process Modeling of Crime', Journal of the American Statistical Association 106(493), 100-108.

Morenoff, J. D., Sampson, R. J. and Raudenbush, S. W. (2001), 'Neighborhood Inequality, Collective Efficacy, and the Spatial Dynamics of Urban Violence*', Criminology 39(3), 517-558.

Papachristos, A. (2009), 'Murder by Structure: Dominance Relations and the Social Structure of Gang Homicide', American Journal of Sociology 115(1), 74-128. 
Papachristos, A., Anthony, B., Piza, E. and Grossman, L. (2016), 'The company you keep? the spillover effects of gang membership on individual gunshot victimization in a co-offending network', Criminology 53, 624-649.

Patel, D. M., Simon, M. A. and Taylor, R. M. (2012), Contagion of Violence - Workshop Summary, Technical report, Institute of Medicine and National Research Council of the National Academies, Washington, D.C.

Petho, A., Fallis, D. S. and Keating, D. (2013), 'Acoustic sensors reveal hidden depth of gun use in D.C.', The Washington Post .

Philadelphia Police Department (2014), Murder/Shooting Analysis 2013, Technical report, Philadelphia Police Department, Philadelphia, PA.

Ramlau-Hansen, H. (1983), 'Smoothing counting process intensities by means of kernel functions', The Annals of Statistics pp. 453-466.

Ratcliffe, J. H. and Rengert, G. F. (2008), 'Near-Repeat Patterns in Philadelphia Shootings', Security Journal 21(1), 58-76.

Reiss, A. J., Roth, J. A., Miczek, K. A. and National Research Council (U. S.). Panel on the Understanding and Control of Violent Behavior (1993), Understanding and preventing violence, National Academy Press-1994, Washington, DC.

Rosenfeld, R., Bray, T. M. and Egley, A. (1999), 'Facilitating Violence: A Comparison of Gang-Motivated, GangAffiliated, and Nongang Youth Homicides', Journal of Quantitative Criminology 15(4), 495-516.

Sallai, J., Hedgecock, W., Volgyesi, P., Nadas, A., Balogh, G. and Ledeczi, A. (2011), 'Weapon classification and shooter localization using distributed multichannel acoustic sensors', Journal of Systems Architecture 57(10), 869-885.

Shalizi, C. R. and Thomas, A. C. (2011), 'Homophily and contagion are generically confounded in observational social network studies', Sociological Methods \& Research pp. 211-329.

Short, M. B., Brantingham, P. J., Bertozzi, A. L. and Tita, G. E. (2010), 'Dissipation and displacement of hotspots in reaction-diffusion models of crime', Proceedings of the National Academy of Sciences 107(9), 3961-3965.

Short, M., Mohler, G., Brantingham, P. J. and Tita, G. (2014), 'Gang Rivalry Dynamics Via Couple Point Process Networks', Discrete and Continuous Dynamical Systems 19(5), 1459-1477.

Skogan, W., Hartnett, S., Bump, N. and Dubois, J. (2009), Evaluation of CeaseFire-Chicago, Technical report, U.S. Department of Justice, Washington, D.C.

Stan Development Team (2016), 'Stan: A c++ library for probability and sampling, version 2.9.0'.

Tarde, G. d. (1903), The Laws of Imitation, H. Holt.

Tita, G. and Cohen, J. (2004), Measuring Spatial Diffusion of Shots Fired Activity, in M. F. Goodchild and D. G. Janelle, eds, 'Spatially Integrated Social Science', Oxford University Press, New York, pp. 171-204.

Tita, G. and Ridgeway, G. (2007), 'The Impact of Gang Formation on Local Patterns of Crime', Journal of Research in Crime and Delinquency 44(2), 208-237.

Tita, G., Riley, J., Ridgeway, G., Grammich, C., Abrahamse, A. and Greenwood, P. (2003), Reducing gun violence: Results from an Intervention in East Los Angeles, RAND Corporation.

Townsley, M., Homel, R. and Chaseling, J. (2003), 'Infectious Burglaries. A Test of the Near Repeat Hypothesis', British Journal of Criminology 43(3), 615-633. 
Webster, D. W., Whitehill, J. M., Vernick, J. S. and Curriero, F. C. (2012), 'Effects of Baltimores Safe Streets Program on Gun Violence: A Replication of Chicagos CeaseFire Program', Journal of Urban Health 90(1), 2740.

Weisburd, D., Groff, E. R. and Yang, S.-M. (2012), The criminology of place: Street segments and our understanding of the crime problem, Oxford University Press.

Wooditch, A. and Weisburd, D. (2015), 'Using space-time analysis to evaluate criminal justice programs: An application to stop-question-frisk practices', Journal of Quantitative Criminology pp. 1-23.

Zimring, F. (1967), 'Is Gun Control Likely to Reduce Violent Killings', University of Chicago Law Review 35, 721. 


\section{A Appendix}

\section{A.1 Hawkes process}

Given a temporal point pattern $t_{1}, \ldots, t_{n} \in \mathcal{R}$, observed on a time window $[0, T]$, the Hawkes process is a socalled self-exciting point process. It has a parsimonious form, parameterized by a conditional intensity function with two parts, a background rate $\mu_{t}(t)$ which may or may not vary with $t$ and a self-excitatory rate which is based only on events which occurred before time $t$ :

$$
\lambda(t)=\mu_{t}(t)+\theta \cdot \sum_{i: t_{i}<t} \omega \cdot \exp \left(-\omega\left(t-t_{i}\right)\right)
$$

Here the triggering kernel is given by an exponential with inverse lengthscale $\omega$ and weight $\theta$, which characterizes the strength and duration of an event's influence on future events. The likelihood of the Hawkes process is simply that of an inhomogeneous Poisson process with intensity $\lambda(t)$ :

$$
\mathcal{L}\left(t_{1}, \ldots, t_{n} \mid \theta, \omega, \mu\right)=\prod_{i} \lambda\left(t_{i}\right) \exp \left(-\int_{t=0}^{T} \lambda(t) d t\right)
$$

To include a spatial dimension, we consider a spatiotemporal point pattern with events at locations $\left(x_{i}, y_{i}, t_{i}\right)$ observed on a window $S \times[0, T]$. We further pick a spatial kernel $k\left(s, s^{\prime}\right)$, and then we have the following conditional intensity:

$$
\lambda(s, t)=\mu(x, y, t)+\theta \sum_{i: t_{i}<t} \omega \cdot \exp \left(-\omega\left(t-t_{i}\right)\right) k\left((x, y),\left(x_{i}, y_{i}\right)\right)
$$

The likelihood is as follows:

$$
\mathcal{L}\left(s_{1}, t_{1}, \ldots, s_{n}, t_{n} \mid \theta, \omega, \mu\right)=\prod_{i} \lambda\left(s_{i}, t_{i}\right) \exp \left(-\int_{S} \int_{t=0}^{T} \lambda(s, t) d s d t\right)
$$

Then we can explicitly calculate the log-likelihood corresponding to Eq. (5):

$$
\begin{aligned}
& \log \mathcal{L}=\sum_{j} \mu\left(x_{j}, y_{j}, t_{j}\right)+\theta \sum_{i: t_{i}<t_{j}} \omega \cdot \exp \left(-\omega\left(t_{j}-t_{i}\right)\right) k\left(\left(x_{j}, y_{j}\right),\left(x_{i}, y_{i}\right)\right) \\
& -\int_{S} \int_{t=0}^{T}\left(\mu(x, y, t)+\theta \sum_{i: t_{i}<t} \omega \cdot \exp \left(-\omega\left(t-t_{i}\right)\right) k\left((x, y),\left(x_{i}, y_{i}\right)\right)\right) d s d t
\end{aligned}
$$

Now for simplicity, we assume that $\int_{S} k\left((x, y),\left(x_{i}, y_{i}\right)\right) d s=1, \forall i$ which we can ensure by properly normalizing the spatial kernel, and also assuming that the lengthscale is much shorter than the size of the spatial domain. We use a Gaussian kernel for $k\left((x, y),\left(x^{\prime}, y^{\prime}\right)\right)$ with lengthscale $\sigma$. Since we are in two dimensions, we have the following normalized kernel:

$$
k\left(s, s^{\prime}\right)=\frac{1}{2 \pi \sigma^{2}} \exp \left(-\frac{\left\|s-s^{\prime}\right\|^{2}}{2 \sigma^{2}}\right)
$$

We estimate the background intensity $\mu(x, y, t)$ using kernel smoothing with an Epanechnikov kernel:

$$
k(d)=\frac{3}{4}\left(1-d^{2}\right) I(|d|<1)
$$

We separately fit $\hat{\mu}_{s}(x, y)$ and $\hat{\mu}_{t}(t)$ and include a weighting term $m_{0}$ to enable the model to downweight this background intensity:

$$
\hat{\mu}(x, y, t)=m_{0} \cdot \hat{\mu}_{s}(x, y) \hat{\mu}_{t}(t)
$$


We performed a sensitivity analysis in Section to investigate the impact of the kernel bandwidth.

In order to learn the parameters of the spatiotemporal Hawkes process, we use Bayesian inference implemented with Markov Chain Monte Carlo methods to find the posterior distribution of the parameters, where we place weakly informative priors Gelman et al. (2008) on them:

- $m_{0} \sim \mathcal{N}_{+}(0,1)$

- $\theta \sim \mathcal{N}_{+}(0,10)$

- $\sigma \sim \mathcal{N}_{+}(0,10)$ where distance is measured in kilometers

- $\omega \sim \mathcal{N}_{+}(0,10)$ where time is measured in $1 /$ days

with $\mathcal{N}_{+}$denoting the Normal distribution truncated to be positive.

We coded our model in the probabilistic programming language Stan Stan Development Team (2016), which implements Hamiltonian Monte Carlo sampling to efficiently explore the posterior distribution ove all of the parameters of our model. We ran 4 chains for 1,000 iterations each. Convergence diagnostics are discussed in Section A.7.

\section{A.2 Dealing with holidays}

As shown in Figure 4 (left), many extra shootings are observed in our dataset on the days before and after New Year's Eve and the Fourth of July. There were 5,653 total shootings on these days, accounting for 36\% of all shootings. We considered these shootings to be "celebratory" and thus removed July 1st-6th and December 29thJanuary 2nd from our dataset for each year. The time series without these extra shootings is shown in Figure 4 (right). We performed a sensitivity analysis to see whether our main findings were robust to including the holidays. The posterior over the lengthscales was slightly longer-19 minutes and 210 meters and the parameter $\theta$ (which characterizes the number of additional shootings we expect to see after a single shooting) was higher: 0.43 (versus 0.13 in our main model) and correspondingly $m_{0}$ the weight placed on the underlying intensity was 0.57 (versus 0.87 in our main model). All of these findings make sense, as the holiday shootings are by their nature spatiotemporally clustered and the model represents this by increasing the weight and spatiotemporal extent of the self-excitatory component.

\section{A.3 Crime Offense Reports Data}

Conventional reported crime data on firearm assaults, as seen in Figure 5, manifests a similar spatial distribution and temporal distribution as AGLS-detected gunshots. However, the intensity levels are markedly different. As a result of these intensity differences between measures, heterogeneity in the intensity of incident clusters is much more visible in the AGLS spatial distribution than the conventional firearm assault distribution. In addition, as seen in the bottom right graph in Figure 5, firearm assault data lags AGLS data by several hours. A lag of several minutes would be consistent with existing knowledge on reporting practices, but a lag of hours likely reflects a recording practice associated with duty shifts or a similar process. For this reason, 911 calls for service data on "shots fired" will provide a better source for estimating spatiotemporal models if AGLS data is not present and binning is observed in publicly reported crimes data.

To verify that our spatiotemporal models could be used on 911 calls for service data, we refit our final model using the 911 calls for service data for "sounds of gunshots" for an overlapping temporal period beginning in 2011 and ending in 2013. The spatial lengthscale was 221 [214,228] meters. The temporal lengthscale was 9 [8.4,9.4] minutes. And the self-exciting paramters, corresponding to the fraction of events attributable to the conditional as opposed to background intensity, was $0.15[0.14,0.16]$. These results closely matched those obtained using AGLS data. The degree of similarity is likely a product of the high fraction of gunshot events reported to police both by civilians and the AGLS system. In cities with more limited reporting or deployment, models fit on different data sources may not yield as consistent results. 

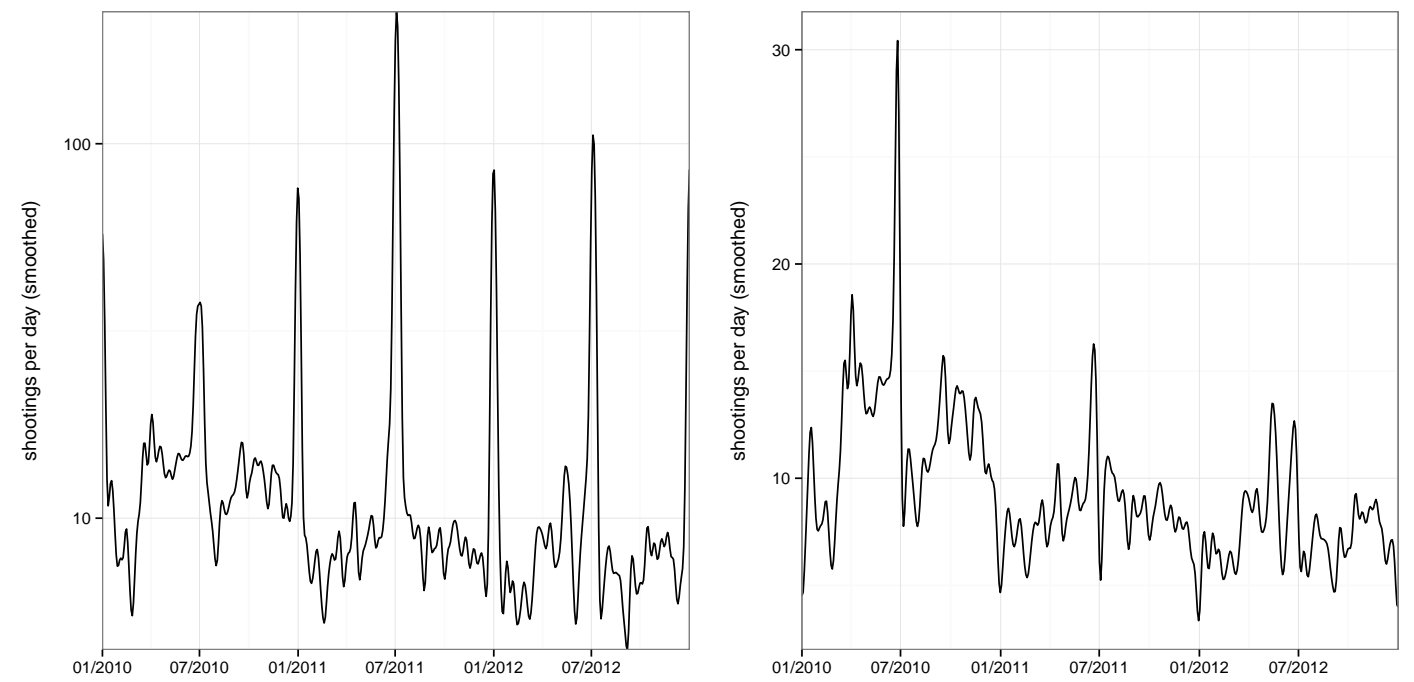

Figure 4: Smoothed time series of shots fired in Washington D.C., 2011, comparing the original data (left) to the data after removing July 1st-6th and December 29th-January 2nd for each year (right).

\section{A.4 Sensitivity analysis for smoothing kernel bandwidth}

We investigate the choice of the bandwidth for the smoothing kernel for estimating the underlying intensity $\hat{\mu}(x, y, t)$. For spatial bandwidths in $[0.5,1,1.6] \mathrm{km}$ and temporal bandwidths in $[0.5,1,7,14]$ days we fit our model using only data from 2011-12. The learned spatial lengthscales were between 130 and 210 meters, matching our main model's posterior (126 meters), while the learned temporal lengthscales were between 12 minutes and 20 minutes, slightly longer than in our main model (10 minutes).

\section{A.5 Sensitivity analysis for inclusion of duplicate events}

In our main analysis, we merged shootings which occurred within 1 minute and 100 meters together, treating them as a single shooting (after also removing holidays). We thus removed 415 shootings which we considered to be duplicates (4\% of our sample). As a sensitivity analysis, we fit our model to the original dataset, without holidays, but with each of the near-repeats included. All of our findings were consistent with our main findings: the lengthscale was 100 meters in space (slightly shorter than the main model) and 11 minutes in time, and $m_{0}$, the weight placed on the endogeneous component was 0.86 , while $\theta$ was 0.14 . This underscores the very local and limited extent to which we are able to find evidence of diffusion in our dataset.

\section{A.6 Samples from the posterior for lengthscale parameters}

Inference for our model for the AGLS data resulted in a posterior concentrated around its mode, as shown in Figure 7.

\section{A.7 Traceplots and convergence diagnostics}

We implemented the model in Section A.1 in the probabilistic programming language Stan Stan Development Team (2016), which uses Hamiltonian Monte Carlo to approximately sample from the posterior of the distribution over the parameters. We ran 4 chains for 1, 000 iterations each, discarding the first 500 draws as burn-in, for a total of 2,000 draws. We show traceplots in Figure 8. The Gelman-Rubin statistic $\hat{R}=1$ for every parameter, 
with effective sample sizes above 1,000 . Thus we conclude that the chains have mixed and converged, and we are sampling from the true posterior of the model. 


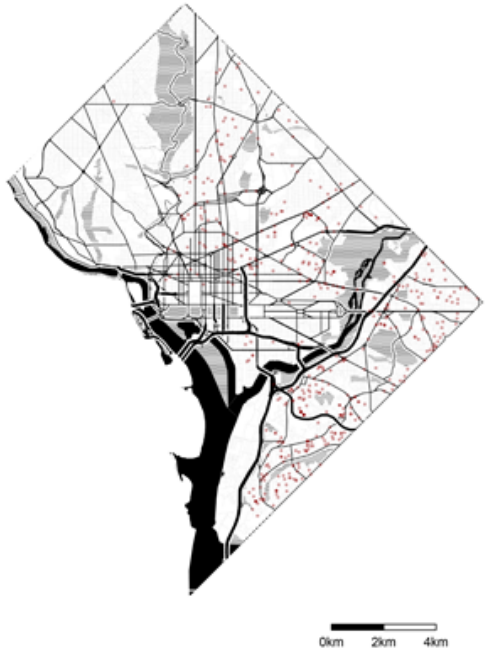

(a)

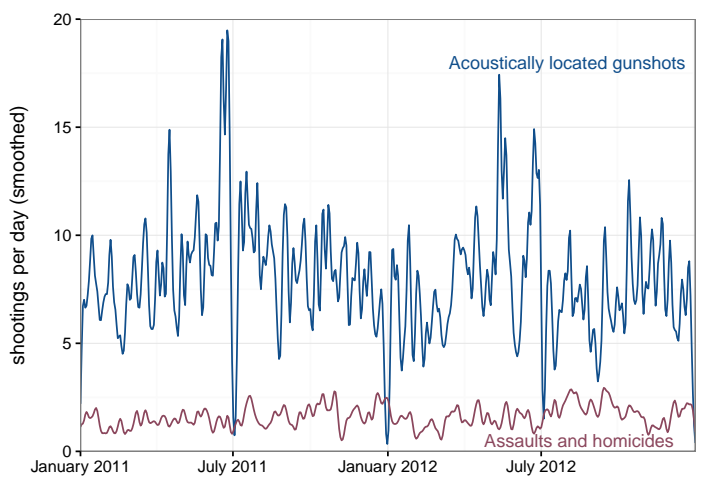

(d)

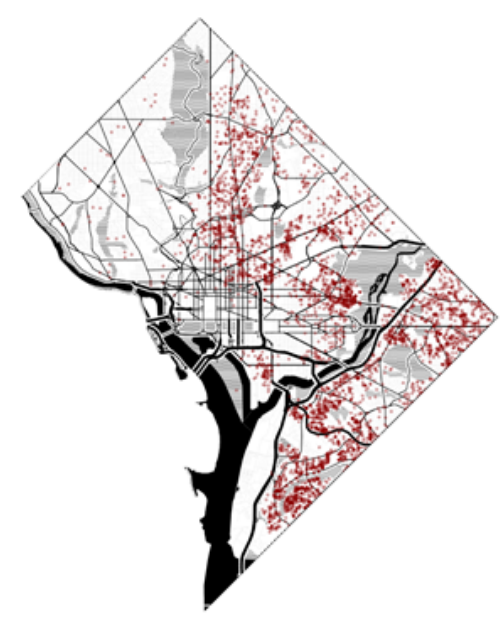

(b)

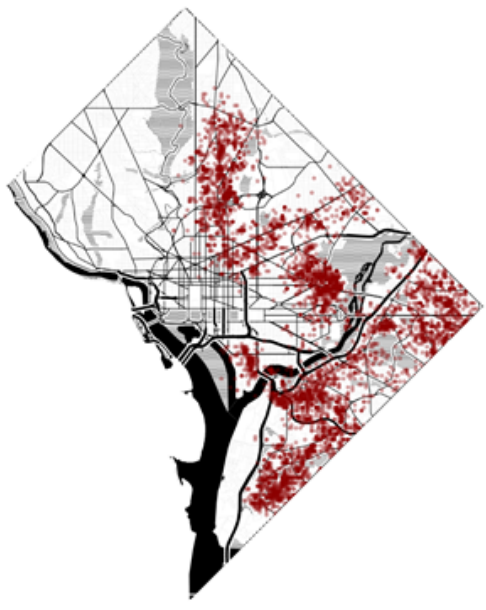

(c)

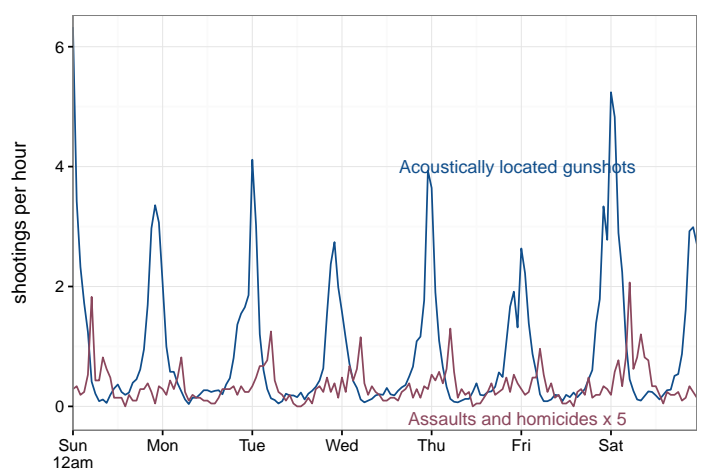

(e)

Figure 5: Spatial and Temporal Distributions of Firearm Assaults and Shots Fired in Washington D.C., 2011. The spatial distribution of aggravated assaults (a), 911 "shots fired" calls (b), and acoustically detected gunshots (c) is shown subfigures (a-c). Monthly and day-of-week temporal distributions are shown in subfigures (d-e). 


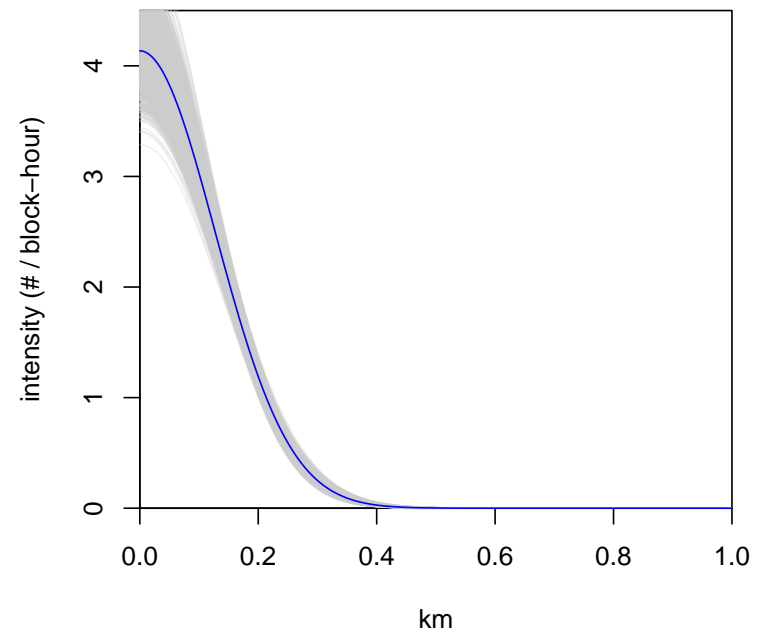

(a) Conditional intensity in space at time $t=0$

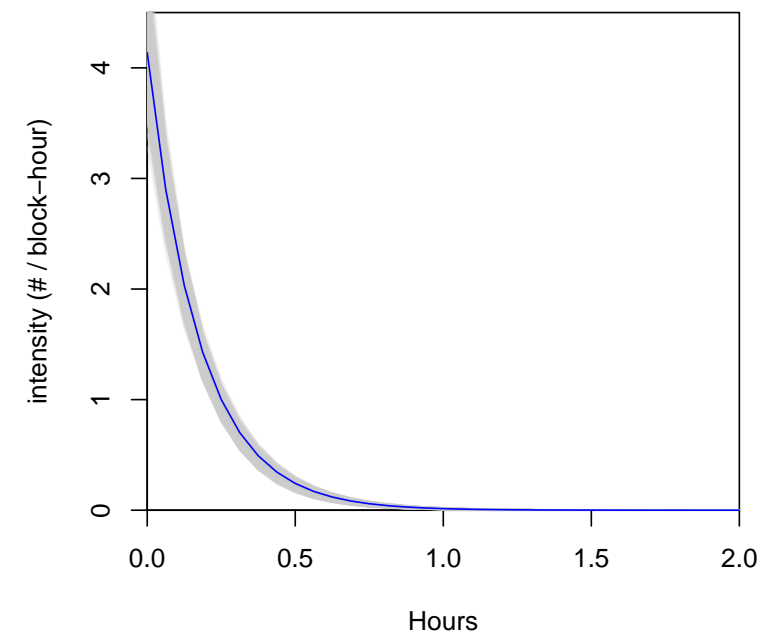

(b) Conditional intensity in time at distance $s=0$

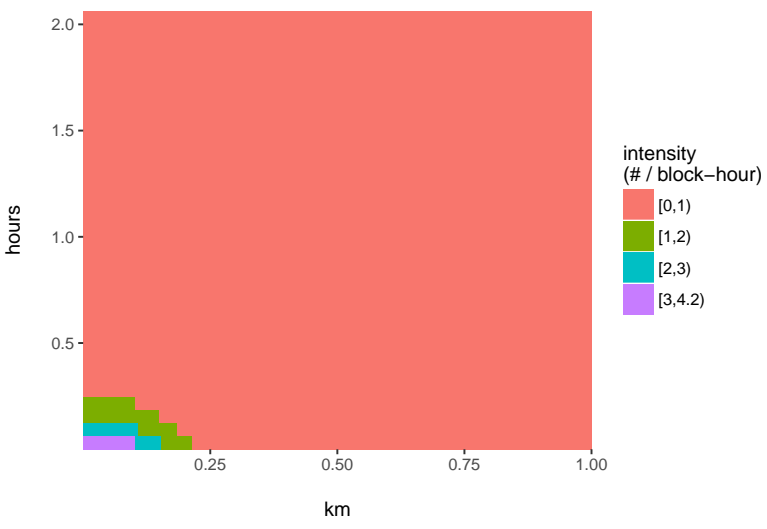

(c) Spatiotemporal conditional intensity

Figure 6: For the 911 "shots fired" calls, we visualize the self-excitatory component of the Hawkes process, corresponding to the product of a spatial and temporal kernel, in space (a) at time $t=0$, in time (b) at distance $s=0$, and in space/time (c). In (a) and (b) the blue line is the mean of the posterior distribution. The gray lines show samples from the posterior distribution. 


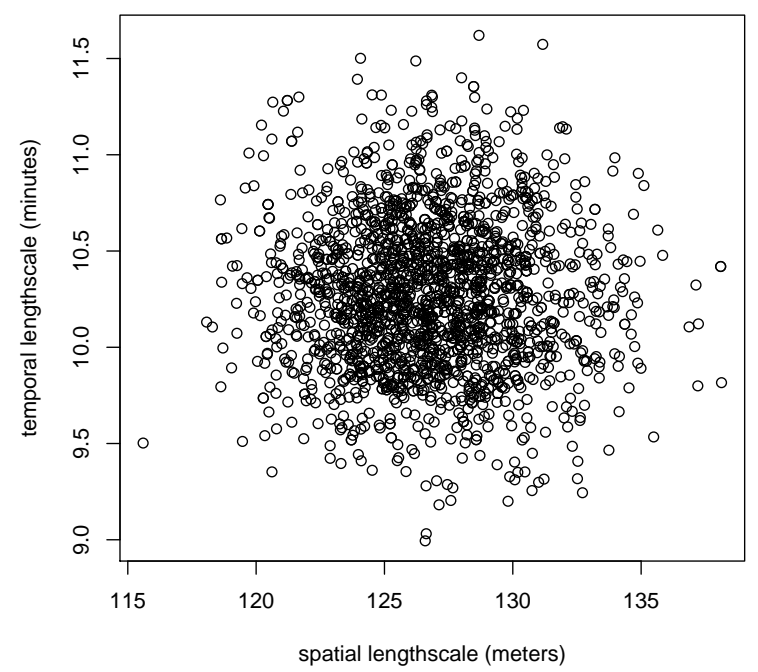

Figure 7: 2000 draws from the posterior of the main model are shown for the temporal and spatial lengthscale parameters. The posterior is concentrated around its mode.
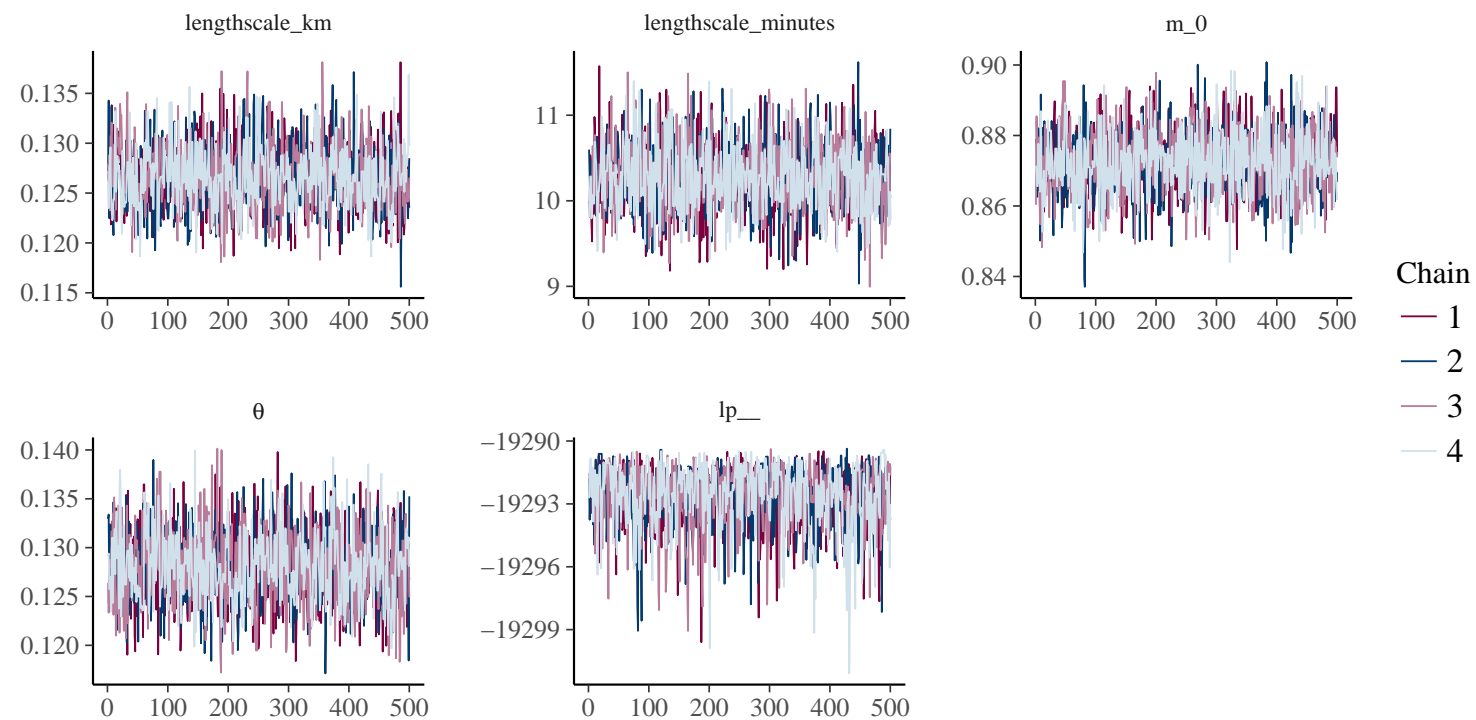

$-3$

Figure 8: Traceplots for the parameters of the model fit with HMC, showing good convergence and mixing. 